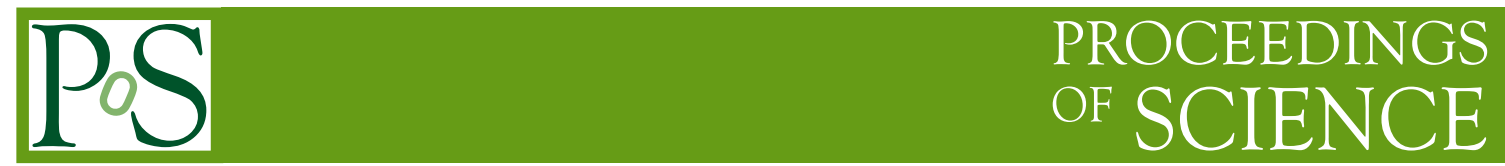

\title{
Status of Light Sterile Neutrinos
}

\author{
Carlo Giunti* \\ INFN, Sezione di Torino, Via P. Giuria 1, I-10125 Torino, Italy \\ E-mail: giunti@to.infn.it
}

The LSND, Gallium and reactor neutrino anomalies can be explained by short-baseline neutrino oscillations due to the mixing of the active neutrinos with sterile neutrinos at the $\mathrm{eV}$ scale. I review the results of a $3+1$ global fit of short-baseline neutrino oscillation data which includes the recent measurements of the MINOS, IceCube, and NEOS experiments.

EPS-HEP2017, 2017 European Physical Society Conference on High Energy Physics, 5-12 July 2017, Venezia, Italy

\footnotetext{
${ }^{*}$ Speaker.
} 
The LSND [1], Gallium [2-5] and reactor [6-8] anomalies are intriguing indications in favor of short-baseline neutrino oscillations due to sterile neutrinos at the $\mathrm{eV}$ scale. Here, I consider $3+1$ active-sterile neutrino mixing (see Ref. [9]), in which there are three sub-eV massive neutrinos $v_{1}$, $v_{2}$, and $v_{3}$ which are the main constituents of the three standard active neutrinos $v_{e}, v_{\mu}, v_{\tau}$, and there is a fourth massive neutrino $v_{4}$ at the $\mathrm{eV}$ scale which is mainly sterile $\left(v_{4} \simeq v_{s}\right)$.

In the framework of $3+1$ active-sterile mixing, short-baseline (SBL) experiments are sensitive only to the oscillations generated by the squared-mass difference $\Delta m_{41}^{2} \simeq \Delta m_{42}^{2} \simeq \Delta m_{43}^{2} \gtrsim 1 \mathrm{eV}^{2}$, with $\Delta m_{j k}^{2} \equiv m_{j}^{2}-m_{k}^{2}$, that is much larger than the the solar squared-mass difference $\Delta m_{\mathrm{SOL}}^{2}=$ $\Delta m_{21}^{2} \approx 7.4 \times 10^{-5} \mathrm{eV}^{2}$ and the atmospheric squared-mass difference $\Delta m_{\mathrm{ATM}}^{2}=\left|\Delta m_{31}^{2}\right| \simeq\left|\Delta m_{32}^{2}\right| \approx$ $2.5 \times 10^{-3} \mathrm{eV}^{2}$, which generate the observed solar, atmospheric and long-baseline neutrino oscillations explained by the standard three-neutrino mixing [10]. The $3+1$ active-sterile mixing scheme is a perturbation of the standard three-neutrino mixing in which the $3 \times 3$ unitary mixing matrix $U$ is extended to a $4 \times 4$ unitary mixing matrix with $\left|U_{e 4}\right|^{2},\left|U_{\mu 4}\right|^{2},\left|U_{\tau 4}\right|^{2} \ll 1$. The effective oscillation probabilities of the flavor neutrinos in short-baseline experiments are given by [11]

$$
P_{\alpha \beta}^{(\mathrm{SBL})} \simeq\left|\delta_{\alpha \beta}-\sin ^{2} 2 \vartheta_{\alpha \beta} \sin ^{2}\left(\frac{\Delta m_{41}^{2} L}{4 E}\right)\right|,
$$

where $\alpha, \beta=e, \mu, \tau, s, L$ is the source-detector distance and $E$ is the neutrino energy. The shortbaseline oscillation amplitudes depend only on the absolute values of the elements in the fourth column of the mixing matrix:

$$
\sin ^{2} 2 \vartheta_{\alpha \beta}=\left.4\left|U_{\alpha 4}\right|^{2}\left|\delta_{\alpha \beta}-\right| U_{\beta 4}\right|^{2} \mid .
$$

Hence, the transition probabilities of neutrinos and antineutrinos are equal and it is not possible to measure in short-baseline experiments any $\mathrm{CP}$-violating effect generated by the complex phases in the mixing matrix. $\mathrm{CP}$ violating effects due to active-sterile neutrino mixing can, however, be observed in long-baseline [12-21] and solar [22] neutrino experiments.

Here I review the results of the $3+1$ global fit of short-baseline neutrino oscillation data presented in Ref. [23], which includes the recent measurements of the MINOS [24], IceCube [25], and NEOS [26] experiments.

It is well known (see Ref. [9]) that the global fits of short-baseline data are affected by the socalled "appearance-disappearance" tension, that is present [27] for any number $N_{s}$ of sterile neutrinos in $3+N_{s}$ mixing schemes which are perturbations of the standard three-neutrino mixing required for the explanation of the observation of solar, atmospheric and long-baseline neutrino oscillations. In Ref. [28], we proposed a "pragmatic approach" in which the appearance-disappearance tension is alleviated by excluding from the global fit the low-energy bins of the MiniBooNE experiment [29], which have an anomalous excess of $\stackrel{(-)}{v_{e}}$-like events that is widely considered to be suspicious because of the large background. Part of the MiniBooNE low-energy anomaly may be explained by taking into account nuclear effects in the energy reconstruction [30,31], but this effect is not sufficient to solve the problem [32]. Some of the low-energy $v_{e}$-like events can be due to photon events which are indistinguishable from $v_{e}$ events in the MiniBooNE liquid scintillator detector. These photons can be generated by the decays of $\pi^{0}$ 's produced by the neutral-current interactions of the

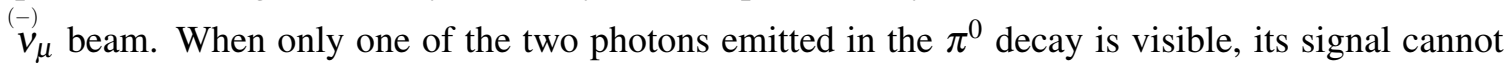




\begin{tabular}{cccc} 
& PrGlo16A & PrGlo16B & PrGlo17 \\
\hline$\chi_{\min }^{2}$ & 262.0 & 530.3 & 595.1 \\
$\mathrm{NDF}$ & 244 & 519 & 579 \\
$\mathrm{GoF}$ & $20 \%$ & $36 \%$ & $31 \%$ \\
$\Delta m_{41}^{2}$ & 1.6 & 1.6 & 1.7 \\
$\left|U_{e 4}\right|^{2}$ & 0.026 & 0.030 & 0.020 \\
$\left|U_{\mu 4}\right|^{2}$ & 0.013 & 0.011 & 0.015 \\
$\sin ^{2} 2 \vartheta_{e \mu}$ & 0.0014 & 0.0013 & 0.0012 \\
$\sin ^{2} 2 \vartheta_{e e}$ & 0.10 & 0.12 & 0.079 \\
$\sin ^{2} 2 \vartheta_{\mu \mu}$ & 0.053 & 0.042 & 0.058 \\
\hline$\Delta \chi_{\mathrm{NO}}^{2}$ & 48.3 & 47.3 & 47.4 \\
$\mathrm{NDF}$ & 3 & 4 & 4 \\
$n \sigma_{\mathrm{NO}}$ & 6.4 & 6.1 & 6.1 \\
\hline$\Delta \chi_{\mathrm{PG}}^{2}$ & 3.8 & 4.7 & 7.2 \\
$\mathrm{NDF}_{\mathrm{PG}}$ & 2 & 2 & 2 \\
$\mathrm{GoF}_{\mathrm{PG}}$ & $15 \%$ & $9.7 \%$ & $2.7 \%$ \\
\hline
\end{tabular}

Table 1: Results of the pragmatic $3+1$ global PrGlo16A, PrGlo16B, and PrGlo17 fits of SBL data. The first group of rows gives: the minimum $\chi^{2}\left(\chi_{\min }^{2}\right)$, the number of degrees of freedom (NDF), the goodness of fit (GoF), the best fit values of the mixing parameters $\Delta m_{41}^{2},\left|U_{e 4}\right|^{2},\left|U_{\mu 4}\right|^{2}$, and of the oscillation amplitudes $\sin ^{2} 2 \vartheta_{e \mu}, \sin ^{2} 2 \vartheta_{e e}, \sin ^{2} 2 \vartheta_{\mu \mu}$. The second group of rows gives the $\chi^{2}$ difference $\Delta \chi_{\mathrm{NO}}^{2}$ between the $\chi^{2}$ of no oscillations and $\chi_{\min }^{2}$ and the resulting number of $\sigma$ 's $\left(n \sigma_{\mathrm{NO}}\right)$ for $\mathrm{NDF}_{\mathrm{NO}}$ degrees of freedom corresponding to the number of fitted parameters. The third group of rows gives the results for the appearance-disappearance parameter goodness of fit [34]: the $\chi^{2}$ difference $\Delta \chi_{\mathrm{PG}}^{2}$ and the resulting goodness of fit $\mathrm{GoF}_{\mathrm{PG}}$ for $\mathrm{NDF}_{\mathrm{PG}}$ degrees of freedom.

be distinguished from a $v_{e}$ event in a liquid-scintillator detector. The suspicion that this photon background may be responsible for the MiniBooNE low-energy excess motivated the realization of the MicroBooNE experiment at Fermilab [33], which is able to distinguish between photon and $\stackrel{(-)}{v_{e}}$ events by using a Liquid Argon Time Projection Chamber (LArTPC). Waiting for the results of this experiment, it is reasonable to adopt the pragmatic approach of omitting from the global fit the MiniBooNE low-energy data. In this paper I assume the pragmatic approach from the beginning.

The pragmatic global fit of short-baseline neutrino oscillation data [23] considers the following three groups of experiments:

(A) The $\stackrel{(-)}{v_{\mu}} \rightarrow \stackrel{(-)}{v_{e}}$ appearance data of the LSND [1], MiniBooNE [29] (without the anomalous low-energy bins), BNL-E776, KARMEN, NOMAD, ICARUS, and OPERA experiments.

(B) The following $\stackrel{(-)}{v}_{e}$ disappearance data: 1) The ratios of measured and predicted [6-8] $\bar{v}_{e}$ rates of the short-baseline reactor experiments listed in Table 1 of Ref. [23]); 2) The $\bar{v}_{e}$ spectra measured in the Bugey-3 and NEOS [26] short-baseline reactor experiments; 3) the data of the GALLEX and SAGE Gallium radioactive source experiments with the statistical method 


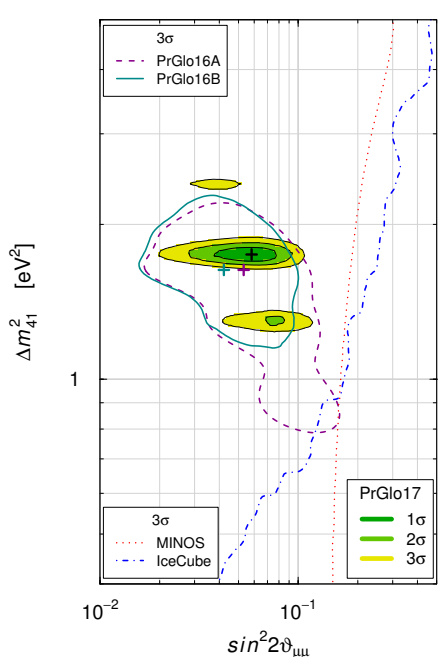

(a)

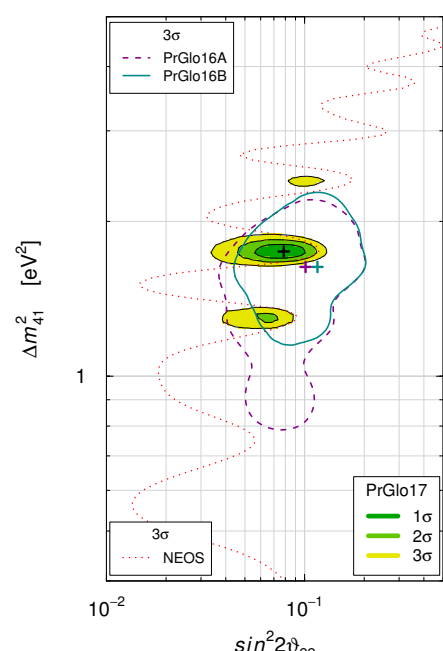

(b)

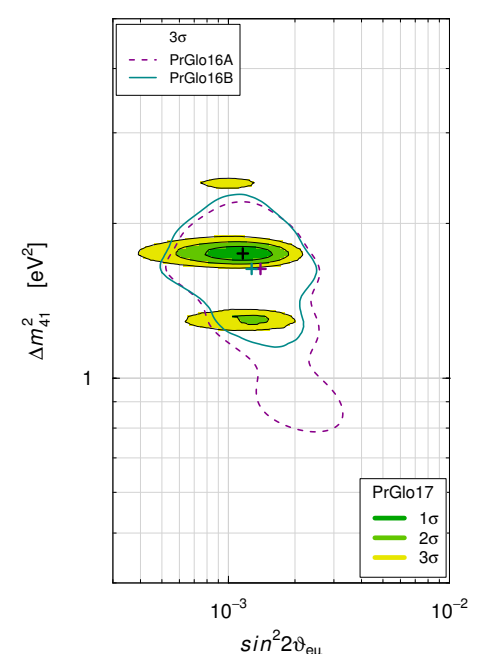

(c)

Figure 1: Allowed regions in the $\sin ^{2} 2 \vartheta_{\mu \mu}-\Delta m_{41}^{2}$ (a), $\sin ^{2} 2 \vartheta_{e e}-\Delta m_{41}^{2}$ (b), and $\sin ^{2} 2 \vartheta_{e \mu}-\Delta m_{41}^{2}$ (c) planes obtained in the pragmatic 3+1 global PrGlo16A, PrGlo16B, and PrGlo17 fits of SBL data.

discussed in Ref. [4]; 4) the solar neutrino constraint on $\sin ^{2} 2 \vartheta_{e e}$ [23]; 5) the KARMEN and LSND $v_{e}+{ }^{12} \mathrm{C} \rightarrow{ }^{12} \mathrm{~N}_{\mathrm{g} . \mathrm{s} .}+e^{-}$scattering data, with the method discussed in Ref. [35].

(C) The constraints on $\stackrel{(-)}{v}_{\mu}$ disappearance obtained from the data of the CDHSW experiment, from the analysis in Ref. [36] of the data of atmospheric neutrino oscillation experiments, from the analysis of the SciBooNE-MiniBooNE neutrino and antineutrino data, and the recent constraints of the MINOS [24] and IceCube [25] experiments.

Table 1 and Figure 1 summarize the results of the following three global fits:

PrGlo16A. In this analysis we considered all the appearance and disappearance SBL data available in 2016, except MINOS [24] and IceCube [25]. The PrGlo16A fit is an update of the PrGLO fit presented in Ref. [9], with a similar set of data.

PrGlo16B. In this analysis we added the MINOS [24] and IceCube [25] data to the data considered in the PrGlo16A fit, in order to clarify their effects on the results of the analysis.

PrGlo17. In this analysis we added the NEOS [26] data, which have been available to us in the beginning of 2017.

From Table 1 one can see that all the three fits have an acceptable goodness of fit and the case of no oscillations is disfavored at the level of about $6 \sigma$. On the other hand, the parameter goodness of fit, decreases from 15\% in the PrGlo16A fit to 9.7\% in the PrGlo16B fit to $2.7 \%$ in the PrGlo17 fit. This is a symptom of the increase of the appearance-disappearance tension caused by the addition of the MINOS and IceCube data in the PrGlo16B fit and the addition of NEOS data in the PrGlo17 fit.

Figure 1(a) shows the allowed regions in the $\sin ^{2} 2 \vartheta_{\mu \mu}-\Delta m_{41}^{2}$ plane, which is relevant for $\stackrel{(-)}{\nu}_{\mu}$ disappearance. One can see that the constraints on $\stackrel{(-)}{\mu}_{\mu}$ disappearance given by the MINOS and 


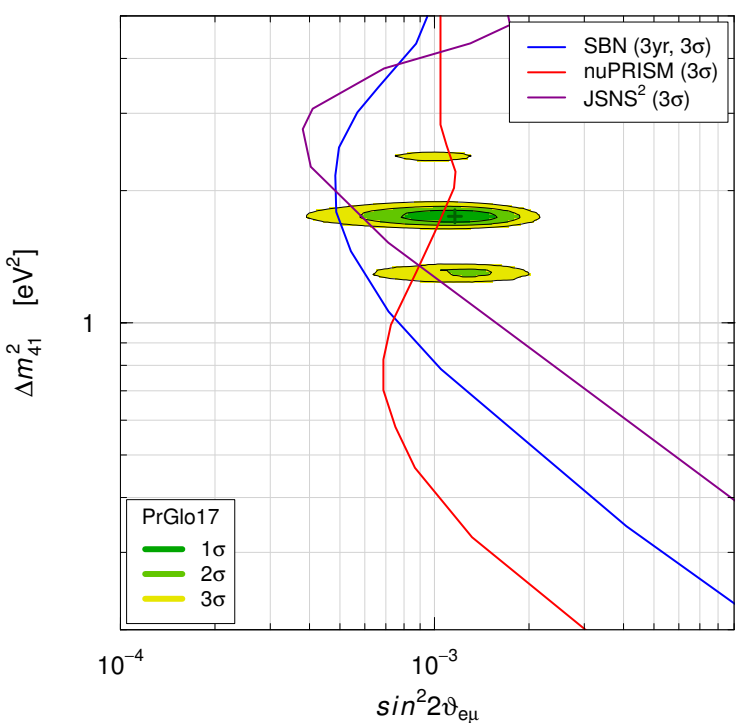

(a)

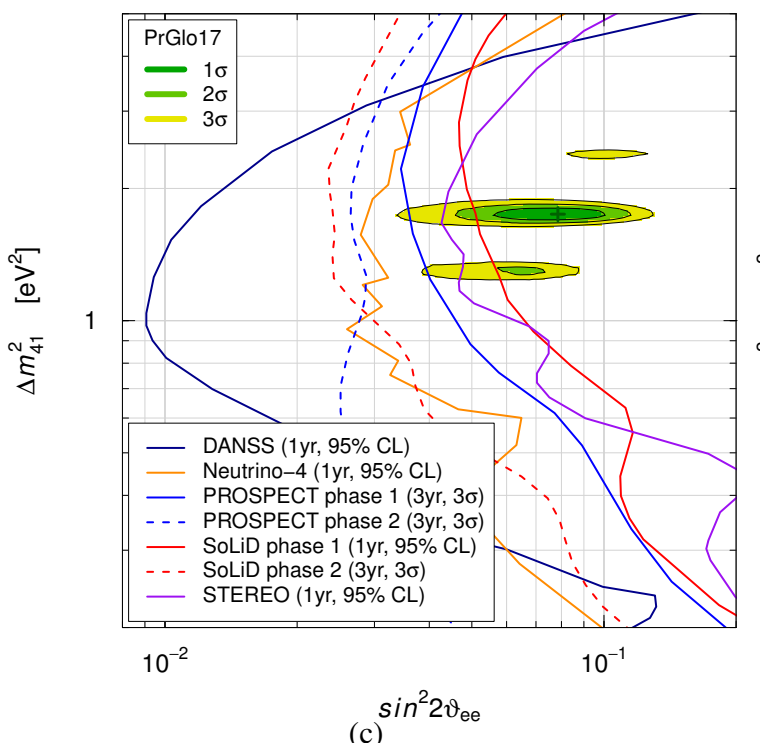

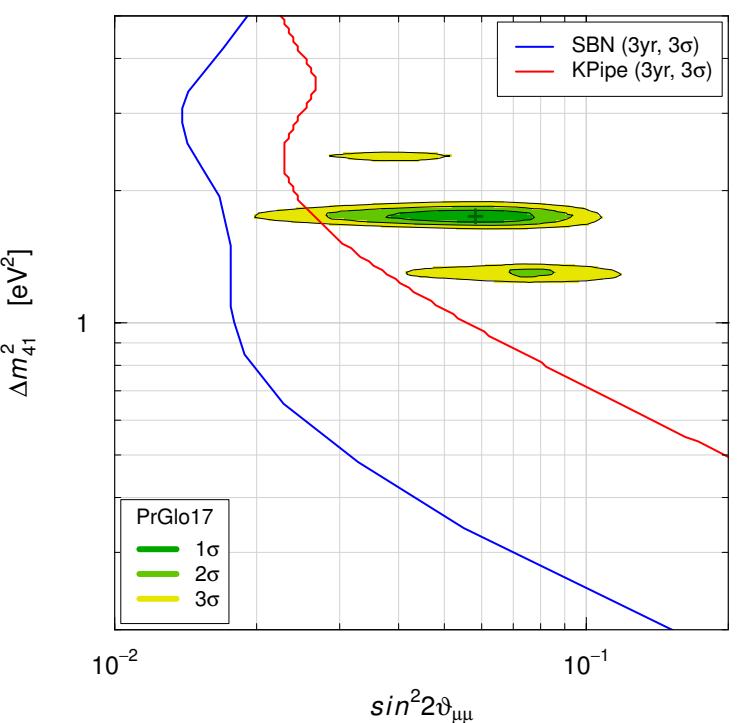

(b)

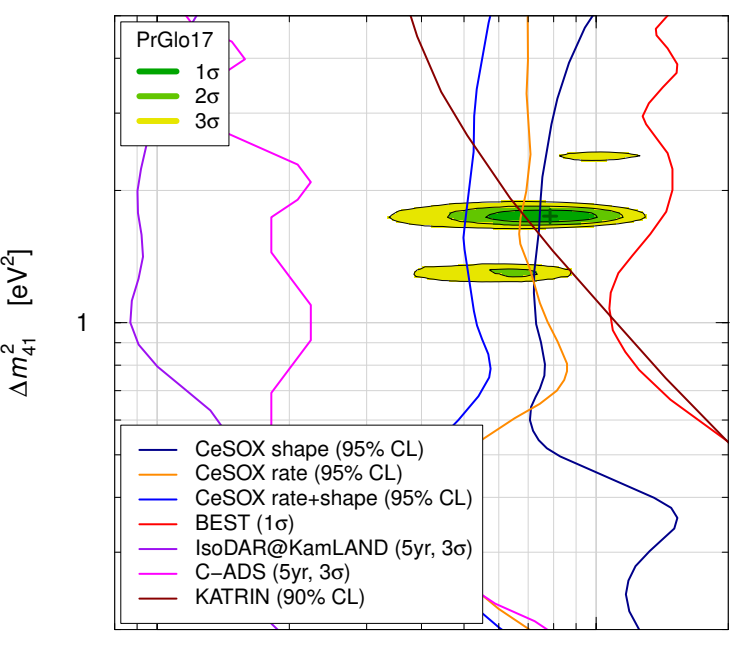

$10^{-1}$

$\sin ^{2} 2 \vartheta_{\mathrm{ee}}$

Figure 2: Sensitivities of future experiments compared with the PrGlo17 allowed regions in Fig. 1.

IceCube data disfavor the low- $\Delta m_{41}^{2}-$ high- $\sin ^{2} 2 \vartheta_{\mu \mu}$ part of the region allowed by the PrGlo16A fit. This effect was expected [37] and is consistent with the results of the $3+1$ global fit presented in Ref. [38], which updated Ref. [39] with the addition of the IceCube data. The decrease of the parameter goodness of fit from 15\% in the PrGlo16A fit to 9.7\% in the PrGlo16B fit is due to the increase of the appearance-disappearance tension caused by the shrinking if the allowed range of $\left|U_{\mu 4}\right|^{2}$ from $0.0050-0.033$ at $3 \sigma$ in the PrGlo16A fit to $0.0048-0.023$ in the PrGlo16B fit.

Figure 1(b) shows the allowed regions in the $\sin ^{2} 2 \vartheta_{e e}-\Delta m_{41}^{2}$ plane, which is relevant for $\stackrel{(-)}{v_{e}}$ disappearance. One can see that the inclusion of the NEOS constraints has the dramatic effect of fragmenting the allowed region in three islands with narrow $\Delta m_{41}^{2}$ widths. The best-fit island is at $\Delta m_{41}^{2} \approx 1.7 \mathrm{eV}^{2}$. There is an island allowed at $2 \sigma$ at $\Delta m_{41}^{2} \approx 1.3 \mathrm{eV}^{2}$, and an island allowed at 
$3 \sigma$ at $\Delta m_{41}^{2} \approx 2.4 \mathrm{eV}^{2}$. Moreover, the NEOS constraints shifts the allowed range of $\left|U_{e 4}\right|^{2}$ from $0.013-0.050$ at $3 \sigma$ in the PrGlo16B fit to $0.0098-0.031$ in the PrGlo17 fit. Therefore, the appearance-disappearance tension is increased, as shown by the decrease of the parameter goodness of fit from $9.7 \%$ in the PrGlo16B fit to $2.7 \%$ in the PrGlo17 fit.

Figure 2 shows a comparison of the sensitivities of future experiments with the PrGlo17 allowed regions for: (a) $\stackrel{(-)}{v_{\mu}} \rightarrow \stackrel{(-)}{v_{e}}$ transitions; (b) $\stackrel{(-)}{v_{\mu}}$ disappearance; (c), (d) $\stackrel{(-)}{v_{e}}$ disappearance. It is clear that these experiments will give definitive information on the existence of active-sterile shortbaseline oscillations connected with the LSND, Gallium and reactor anomalies.

In conclusion, the comparison of the PrGlo16A and PrGlo16B fits shows that, as expected [37, 38], the MINOS and IceCube constraints on $v_{\mu}$ disappearance disfavor the low- $\Delta m_{41}^{2}$-high-sin $2 \vartheta_{\mu \mu}$ and the low- $\Delta m_{41}^{2}-$ high- $\sin ^{2} 2 \vartheta_{e \mu}$ parts of the allowed region. The addition of the NEOS data in the PrGlo17 fit has the more dramatic effect of reducing the allowed region to three islands with narrow $\Delta m_{41}^{2}$ widths and $0.00048 \lesssim \sin ^{2} 2 \vartheta_{e \mu} \lesssim 0.0020$ at $3 \sigma$. The best-fit island is at $\Delta m_{41}^{2} \approx 1.7 \mathrm{eV}^{2}$. There is an island allowed at $2 \sigma$ at $\Delta m_{41}^{2} \approx 1.3 \mathrm{eV}^{2}$, and an island allowed at $3 \sigma$ at $\Delta m_{41}^{2} \approx 2.4 \mathrm{eV}^{2}$. However, as illustrated in Fig. 2, the ongoing and planned experiments have the possibility to cover all the allowed regions of the mixing parameters and we expect that they will reach in a few years a definitive conclusion on the existence of the light sterile neutrinos that generate the short-baseline oscillations indicated by the LSND experiment and by the Gallium and reactor neutrino anomalies.

Let me finally emphasize that the confirmation of the existence of sterile neutrinos would be a major discovery which would have a profound impact not only on neutrino physics, but on our whole view of fundamental physics, because it would prove that there is new physics beyond the Standard Model at the low-energies accessible in laboratory experiments. The measurement of the properties of the sterile neutrinos can give important information on this new physics (see Refs. [40,41]).

\section{References}

[1] LSND, A. Aguilar et al., Phys. Rev. D64 (2001) 112007, hep-ex/0104049.

[2] M. Laveder, Nucl. Phys. Proc. Suppl. 168 (2007) 344.

[3] C. Giunti and M. Laveder, Mod. Phys. Lett. A22 (2007) 2499, hep-ph/0610352.

[4] C. Giunti and M. Laveder, Phys. Rev. C83 (2011) 065504, arXiv:1006.3244.

[5] C. Giunti et al., Phys. Rev. D86 (2012) 113014, arXiv:1210.5715.

[6] T.A. Mueller et al., Phys. Rev. C83 (2011) 054615, arXiv:1101.2663.

[7] G. Mention et al., Phys. Rev. D83 (2011) 073006, arXiv:1101.2755.

[8] P. Huber, Phys. Rev. C84 (2011) 024617, arXiv:1106.0687.

[9] S. Gariazzo et al., J. Phys. G43 (2016) 033001, arXiv:1507.08204.

[10] Particle Data Group, C. Patrignani et al., Chin. Phys. C40 (2016) 100001.

[11] S.M. Bilenky, C. Giunti and W. Grimus, Eur. Phys. J. C1 (1998) 247, hep-ph/9607372.

[12] A. de Gouvea, K.J. Kelly and A. Kobach, Phys. Rev. D91 (2015) 053005, arXiv:1412.1479.

[13] N. Klop and A. Palazzo, Phys. Rev. D91 (2015) 073017, arXiv:1412.7524. 
[14] J.M. Berryman et al., Phys. Rev. D92 (2015) 073012, arXiv:1507.03986.

[15] R. Gandhi et al., JHEP 11 (2015) 039, arXiv:1508.06275.

[16] A. Palazzo, Phys.Lett. B757 (2016) 142, arXiv:1509.03148.

[17] S.K. Agarwalla et al., JHEP 02 (2016) 111, arXiv:1601.05995.

[18] S.K. Agarwalla, S.S. Chatterjee and A. Palazzo, JHEP 1609 (2016) 016, arXiv:1603.03759.

[19] S. Choubey and D. Pramanik, Phys.Lett. B764 (2017) 135, arXiv:1604.04731.

[20] S.K. Agarwalla, S.S. Chatterjee and A. Palazzo, Phys. Rev. Lett. 118 (2017) 031804, arXiv:1605.04299.

[21] F. Capozzi et al., Phys.Rev. D95 (2017) 033006, arXiv:1612.07764.

[22] H. Long, Y. Li and C. Giunti, Phys. Rev. D 87, 113004 (2013) 113004, arXiv:1304.2207.

[23] S. Gariazzo et al., JHEP 1706 (2017) 135, arXiv:1703.00860.

[24] MINOS, P. Adamson et al., Phys. Rev. Lett. 117 (2016) 151803, arXiv:1607.01176.

[25] IceCube, M.G. Aartsen et al., Phys. Rev. Lett. 117 (2016) 071801, arXiv:1605.01990.

[26] NEOS, Y. Ko et al., Phys.Rev.Lett. 118 (2017) 121802, arXiv:1610.05134.

[27] C. Giunti and E.M. Zavanin, Mod. Phys. Lett. A31 (2016) 1650003, arXiv:1508.03172.

[28] C. Giunti et al., Phys. Rev. D88 (2013) 073008, arXiv:1308.5288.

[29] MiniBooNE, A. Aguilar-Arevalo et al., Phys. Rev. Lett. 110 (2013) 161801, arXiv:1303.2588.

[30] M. Martini, M. Ericson and G. Chanfray, Phys. Rev. D85 (2012) 093012, arXiv:1202.4745.

[31] M. Martini, M. Ericson and G. Chanfray, Phys. Rev. D87 (2013) 013009, arXiv:1211.1523.

[32] M. Ericson et al., Phys. Rev. D93 (2016) 073008, arXiv:1602.01390.

[33] MicroBooNE, S. Gollapinni, arXiv:1510.04412.

[34] M. Maltoni and T. Schwetz, Phys. Rev. D68 (2003) 033020, hep-ph/0304176.

[35] C. Giunti and M. Laveder, Phys. Lett. B706 (2011) 200, arXiv:1111.1069.

[36] M. Maltoni and T. Schwetz, Phys. Rev. D76 (2007) 093005, arXiv:0705.0107.

[37] C. Giunti, arXiv:1609.04688.

[38] G. Collin et al., Phys. Rev. Lett. 117 (2016) 221801, arXiv:1607.00011.

[39] G.H. Collin et al., Nucl. Phys. B908 (2016) 354, arXiv:1602.00671.

[40] R.R. Volkas, Prog. Part. Nucl. Phys. 48 (2002) 161, hep-ph/0111326.

[41] R.N. Mohapatra and A.Y. Smirnov, Ann. Rev. Nucl. Part. Sci. 56 (2006) 569, hep-ph/0603118. 